\title{
Upregulation of PTGFRN in Hepatocellular Carcinoma Predicts Poor Prognosis: A Study Based on the TCGA Database
}

\section{Yanlin Tan}

Guangxi Medical University

\section{Fan Wu}

Guangxi Medical University

Wenzhen Lin

Guangxi Medical University

\section{Yuqin Wei}

Guangxi Medical University

Yitong Lin

Guangxi Medical University

Naixia Chao

Guangxi Medical University

Qingniao Zhou ( $\sim$ ebirdie123@126.com )

Guangxi Medical University https://orcid.org/0000-0002-4804-9415

\section{Research}

Keywords: Prostaglandin F2 Receptor Inhibitor (PTGFRN), HCC, TCGA, prognosis, GSEA

Posted Date: September 15th, 2021

DOI: https://doi.org/10.21203/rs.3.rs-855947/v1

License: (영 This work is licensed under a Creative Commons Attribution 4.0 International License. Read Full License 


\section{Abstract}

Background. The importance of prostaglandin F2 receptor inhibitors (PTGFRN) in the progression of a variety of malignant tumors has been recognized in recent years. So far, no role of PTGFRN in hepatocellular carcinoma (HCC) has been reported. In this study, we focused on the possible mechanisms of PTGFRN in HCC based on the Cancer Genome Atlas (TCGA) data.

Methods. The mRNA gene expression data of PTGFRN were downloaded from TCGA database to analyze the expression level of PTGFRN in HCC. According to the human protein atlas database, the expression difference of PTGFRN protein between HCC and adjacent tissues was verified. Wilcoxon signed-rank test and logistic regression were used to analyze the relationship between PTGFRN and clinicopathological characteristics. Kaplan Meier method and Cox proportional hazards model were used to explore the prognostic role of PTGFRN in HCC. The ROC curve was used to evaluate the diagnostic value of PTGFRN in HCC. Gene Set Enrichment Analysis (GSEA) was used to investigate the function of PTGFRN related Gene sets. Finally, obtain the co-expressed genes of PTGFRN through the cBioPortal database, and use the Kyoto Encyclopedia of Genes and Genomes (KEGG) and Gene Ontology (GO) function enrichment analysis to further explore the role of PTGFRN in HCC regulated related pathways.

Results. Analysis of mRNA expression data of 377 HCC patients showed that the expression of PTGFRN was up-regulated in HCC, which was confirmed by immunohistochemistry. The overexpression of PTGFRN was significantly correlated with clinical stage $(P=0.028)$ and histological grade $(P=0.027)$. High expression of PTGFRN was associated with poorer overall survival.. Meanwhile, multivariate Cox analysis showed that PTGFRN may be a potential independent risk factor for HCC. GSEA enrichment results showed that the upregulated PTGFRN phenotype was concentrated in "endocytosis", "oocyte meiosis" and "ERBB signaling pathway". In addition, through the analysis of KEGG and GO pathways, we found that PTGFRN co-expressed genes are mainly involved in extracellular matrix tissue, epithelial-mesenchymal transition, cell adhesion and cell cycle, and PI3K-Akt/NF-kB signaling pathways.

Conclusions. PTGFRN is highly expressed in $\mathrm{HCC}$ and can be used as an independent predictor of the clinical prognosis of HCC.

\section{Introduction}

Hepatocellular carcinoma (HCC) is one of the most lethal malignant tumors in the world and the second leading cause of cancer-related death [1,2]. And Guangxi Province in China is one of the high-incidence areas of HCC [3]. Due to the high heterogeneity and complex etiology of HCC, it is difficult to predict the prognosis of tumors [4].

In recent years, progress has been made in the early diagnosis and treatment of HCC, which has effectively improved the prognosis of patients [5]. However, due to the high recurrence rate and high metastasis rate, the survival of HCC patients is still facing challenges $[6,7]$. Traditional prognostic models, such as tumor lymph node metastasis (TNM) stage and vascular infiltration, are not ideal for prediction due to the extreme heterogeneity of $\mathrm{HCC}[8,9]$. In addition, biomarkers and histological classification, such as CD34 and AFP, 
etc., are widely used clinically to evaluate the prognosis of HCC patients. But the prognostic effect is limited by sample size, validation bias and other factors $[1,10]$. Thus, new specific molecular markers are urgently needed to improve the prognosis and treatment of patients.

With the rapid development of genome sequencing technology, more and more evidence show that molecular markers have a great contribution to the diagnosis and prognosis prediction of HCC [8, 11]. Prostaglandin F2 Receptor Inhibitor (PTGFRN, also known as CD9P-1, FPRP, EWIF) is a cell surface immune globulin (Ig) superfamily protein that has an effect on angiogenesis and tumor growth in vivo [12]. Its Ig domains include CD101, EWI-2 and IgSF3 [13]. Moreover, PTGFRN can act as a scaffold protein to bind to other proteins in TEM and regulate downstream signaling pathways [14]. Up to now, more and more evidence has shown that PTGFRN is significantly associated with a variety of cancer types and has different expressions and functions in different tumors. For example, Colin et al. [12] found that the expression level of PTGFRN is positively correlated with lung cancer metastasis. Guilmain et al. [15] found that the up-regulation of PTGFRN expression is closely related to the poor prognosis of patients with renal cell carcinoma. Brittany Aguila et al. $[14,16]$ showed that inhibiting the expression of PTGFRN gene can significantly down-regulate the PI3-K/AKT signaling pathway, which affects the survival of solid tumor cells. S Colin et al. [12] found that the high expression of PTGFRN could negatively affect the growth and metastasis of solid tumors. In addition, inhibition of PTGFRN can reduce DNA damage perception and repair, increasing the sensitivity of glioblastoma multiforme (GBM) cells to radiotherapy and chemotherapy [14]. In conclusion, we can see that PTGFRN is related to tumor metastasis, important signaling pathways and so on. However, there is no report about PTGFRN in HCC, and its diagnostic effect and molecular mechanism remain unclear.

In addition, multiple family members of PTGFRN have also played an important role in the development of HCC. Ricardo et al. found that the increased expression and activity of prostaglandin reductase 1 (PTGR1) was often closely related to the progression of HCC [17]. Min Zhang et al. found that the expression level of prostaglandin E2 (PGE2) in HCC cells was significantly higher than that in normal liver cells. PGE2 can activate the EGFR/Akt/NF-KB pathway, promote Snail expression, and thus accelerate the migration and invasion of HCC [18]. Ricard et al. found that high level of 8-EPI-prostaglandin F2a (8-EPI-PGF2a) was positively associated with the risk of $\mathrm{HCC}$ and could be used as a biomarker to diagnose people at high risk of HCC [19]. However, the potential role of PTGFRN in HCC remains to be clarified.

In this study, we conducted the first bioinformatics analysis of PTGFRN expression in HCC, and discussed the correlation between PTGFRN expression and clinical features and prognosis. We also evaluated the effect of PTGFRN on overall and relapse-free survival. GSEA was used to further understand the regulatory network of PTGFRN involved in biological pathways of disease in HCC. PTGFRN co-expressed genes were screened by cBioPortal database, and they were analyzed by KEGG and GO for annotation, visualization and integrated discovery (DAVID) to analyze the potential function of PTGFRN in HCC. Our results demonstrate the important role of PTGFRN in the prognosis of HCC, which may provide a potential target and strategy for the diagnosis and prognosis of HCC.

\section{Results}




\section{Patient characteristics}

Based on the data of TCGA hepatocellular carcinoma, the clinicopathological features and gene expression data of 377 primary tumors in August 2020 were retrospectively analyzed, the details are shown in Table 1. The median age of patients at diagnosis was 59 years (range 16-90 years). In the study cohort, $49.6 \%$ were white, $42.7 \%$ were Asian, and less than $8 \%$ were African American/American Indian. The cancer status included 374 cases with tumor ( $88.2 \%$ ) and 50 cases tumor-free (11.8\%). Stage I, II, III, and IV patients comprised 175 (46.4\%), 87 (23.1\%), $86(22.8 \%)$ and $5(1.3 \%)$ respectively. 
Table 1

Clinical characteristics of TCGA LIHC patients $(\mathrm{n}=$ 377)

\begin{tabular}{|c|c|c|}
\hline Clinical characteristics & Total $(n=377)$ & $(\%)$ \\
\hline \multicolumn{3}{|l|}{ Age } \\
\hline$<60$ & 172 & 45.6 \\
\hline$\geq 60$ & 204 & 54.1 \\
\hline NA & 1 & 0.3 \\
\hline \multicolumn{3}{|l|}{ Vital status } \\
\hline Dead & 132 & 35.0 \\
\hline Alive & 244 & 64.7 \\
\hline NA & 1 & 0.3 \\
\hline \multicolumn{3}{|l|}{ Gender } \\
\hline Female & 122 & 32.4 \\
\hline Male & 255 & 67.6 \\
\hline \multicolumn{3}{|l|}{ Histologic grade } \\
\hline $\mathrm{G} 1-2$ & 235 & 62.3 \\
\hline $\mathrm{G} 3-4$ & 137 & 36.3 \\
\hline NA & 5 & 1.3 \\
\hline \multicolumn{3}{|l|}{ Stage } \\
\hline$|-| \mid$ & 262 & 69.5 \\
\hline III-IV & 91 & 24.1 \\
\hline NA & 24 & 6.4 \\
\hline \multicolumn{3}{|l|}{ Lymph nodes } \\
\hline NO & 257 & 68.2 \\
\hline N1-3 & 4 & 1.0 \\
\hline $\mathrm{Nx}$ & 115 & 30.5 \\
\hline NA & 1 & 0.3 \\
\hline \multicolumn{3}{|l|}{ Distant metastasis } \\
\hline MO & 272 & 72.1 \\
\hline M1 & 4 & 1.1 \\
\hline
\end{tabular}




\section{\begin{tabular}{|l|lll|}
\hline Clinical characteristics & Total $(n=377)$ & $(\%)$ \\
\hline Mx & 101 & 26.8 \\
\hline
\end{tabular}}

\section{High expression of PTGFRN in HCC}

The TCGA data set provides support for our research on the expression level of PTGFRN in LIHC. Through the differential expression scatter plot and paired differential analysis, RNA-seq data from the TCGA-LIHC cohort indicated that PTGFRN expression was significantly increased in HCC tissues $(n=374 ; p=7.147 e-14$; compared with normal liver tissues $(n=50)$, as shown in Fig. 1A. The expression of PTGFRN in paired cancer tissues is also highly statistically significant $(P=1.653 \mathrm{e}-09)$, as shown in Fig. 1B. Then, the expression level of PTGFRN protein in HCC and adjacent tissues was verified by immunohistochemical analysis. Compared with adjacent tissues, the expression level of PTGFRN in HCC tissues was significantly increased in density and intensity (Fig. 1C.D).

\section{Correlation of PTGFRN gene expression on survival and multivariate analysis}

We performed relevant analyses to evaluate the effect of PTGFRN on the prognosis of patients with HCC. As shown in Fig. 2A, the ROC curve of PTGFRN was executed, and the area under the curve (AUC) was 0.719, indicating moderate diagnostic ability. The subgroup analysis indicated that high expression of PTGFRN is significantly different from clinical-stage $(P=0.028)$ and histological type $(P=0.027)$ (Fig. 2B.C). However, the correlation with distant metastasis $(P=0.696)$ and lymph node metastasis $(P=0.232)$ was not significant (Fig. 2D.E). We plotted Kaplan-Meier curves for OS and DFS based on gene expression. As shown in Fig. 3A.B survival analysis, the increase of PTGFRN expression was significantly associated with decreased OS and DFS in patients with liver cancer. The prognosis of LIHC patients with high PTGFRN expression was worse than that of LIHC patients with low PTGFRN expression $(P=0.0048)$. Univariate analysis of the variables listed in Table 2 showed that PTGFRN-high was significantly associated with poor OS (hazard ratio [HR]: 1.343; $95 \%$ confidence interval [Cl]: 1.056-1.707; $P=0.016$ ). Other clinicopathological variables related to poor survival include clinical stage and pathology $M$. The significant risk factors identified in univariate analysis were used in multivariate Cox analysis and finally found that the expression of PTGFRN (HR $=1.277$, $P=0.045$ ) may be a potential independent risk factor for HCC (Table 3). Forest plot analysis (Fig. 3C) showed that clinical stage $(P<0.001)$ and PTGFRN expression $(P=0.045)$ were statistically significant with the prognosis of HCC patients. and the expression of PTGFRN $(P=0.045)$ and the outcome of LIHC patients. In general, PTGFRN can be used as a reliable and effective independent prognostic factor. 
Table 2

Univariate Cox regression analysis of overall survival in TCGA LIHC patients.

\begin{tabular}{|llll|}
\hline Clinicopathologic variable & HR & HR $(95 \%$ Cl) & $\boldsymbol{P}$ \\
\hline Age (continuous) & 1.005 & $0.987-1.023$ & 0.591 \\
\hline Gender (male/female) & 0.780 & $0.487-1.249$ & 0.301 \\
\hline PTGFRN expression & 1.343 & $1.056-1.707$ & 0.016 \\
\hline Grade (G1/G2/G3/G4/Gx & 1.017 & $0.746-1.387$ & 0.914 \\
\hline Stage (I/II/III/IV) & 1.865 & $1.456-2.388$ & $\mathbf{0 . 0 0 0}$ \\
\hline Lymph nodes & 2.022 & $0.494-8.276$ & 0.328 \\
\hline (positive/negative) & 3.850 & $1.207-12.281$ & $\mathbf{0} 023$ \\
\hline Bold values indicate statistically significant, $P<0.05$. & \\
\hline
\end{tabular}

Table 3

Multivariate Cox regression analysis of overall survival in TCGA LIHC patients.

\begin{tabular}{|llll|}
\hline Clinicopathologic variable & HR & HR $(95 \%$ Cl) & $P$ \\
\hline Age (continuous) & 1.008 & $0.988-1.027$ & 0.441 \\
\hline Gender (male/female) & 1.074 & $0.640-1.803$ & 0.788 \\
\hline PTGFRN expression & 1.277 & $1.006-1.621$ & 0.045 \\
\hline Grade (G1/G2/G3/G4/Gx) & 1.068 & $0.765-1.490$ & 0.700 \\
\hline Stage (I/II/III/IV) & 1.889 & $1.429-2.497$ & 0.000 \\
\hline Lymph nodes & 0.741 & $0.167-3.293$ & 0.693 \\
\hline (positive/negative) & 0.941 & $0.257-3.441$ & 0.927 \\
\hline Bold values indicate statistically significant, $P<0.05$. & \\
\hline
\end{tabular}

To explore the potential mechanism of PTGFRN affecting the occurrence of hepatocellular carcinoma, we used gene set enrichment analysis to compare the differential regulation pathways between the high and low expression groups of PTGFRN. Results with significant differences (NOM $P<0.05, \mathrm{FDR}<0.25)$ in the enrichment of MSigDB Collection (c2.cp.kegg.v7.2.symbols.gmt) (Table 4). The Fig. 4 and Fig. 5A-F shows that "endocytosis", "oocyte meiosis", "cancer pathways", "purine metabolism", "renal cell carcinoma", "apoptosis", "glioma" and "ERBB signaling pathway", "ubiquitin-mediated proteolysis", "focal adhesion", "jak stat", "axon guidance", "apoptosis" and "phosphatidylinositol" were significantly enriched in PTGFRN high expression phenotype. 
Table 4

Genome enrichment of GSEA high phenotype.

\begin{tabular}{|llccc|}
\hline $\begin{array}{l}\text { MSigDB } \\
\text { collection }\end{array}$ & Gene set name & NES & $\begin{array}{c}\text { NOMp- } \\
\text { val }\end{array}$ & $\begin{array}{l}\text { FDR } \\
\text { q-val }\end{array}$ \\
\hline $\begin{array}{l}\text { c2.cp.kegg. } \\
\text { v6.2.symbols.gmt }\end{array}$ & KEGG_ENDOCYTOSIS & 2.203 & 0.000 & 0.000 \\
& KEGG_OOCYTE_MEIOSIS & 2.146 & 0.000 & 0.001 \\
& KEGG_PATHWAYS_IN_CANCER & 2.156 & 0.000 & 0.001 \\
& KEGG_PURINE_METABOLISM & 2.164 & 0.000 & 0.001 \\
& KEGG_RENAL_CELL_CARCINOMA & 2.230 & 0.000 & 0.000 \\
& KEGG_GLIOMA & 2.129 & 0.000 & 0.000 \\
& KEGG_APOPTOSIS & 2.053 & 0.000 & 0.002 \\
& KEGG_ERBB_SIGNALING_PATHWAY & 2.107 & 0.000 & 0.000 \\
& KEGG_AXON_GUIDANCE & 2.098 & 0.000 & 0.001 \\
\hline & KEGG_JAK_STAT_SIGNALING_PATHWAY & 2.091 & 0.000 & 0.001 \\
\hline & KEGG_UBIQUITIN_MEDIATED_PROTEOLYSIS & 2.082 & 0.000 & 0.001 \\
\hline KEGG_FOCAL_ADHESION & 2.082 & 0.000 & 0.001 \\
\hline KEGG_SMALL_CELL_LUNG_CANCER & 2.081 & 0.000 & 0.001 \\
\hline KEGG_PHOSPHATIDYLINOSITOL_SIGNALING_SYSTEM & 2.063 & 0.000 & 0.002 \\
\hline Gene sets with NOM & P<0.05 and FDR q<0.25 are considered as significant. & & \\
\hline
\end{tabular}

\section{GO analysis and KEGG analysis of PTGFRN co-expressed genes}

Genes with similar expression patterns may be linked with some functions. In order to explore the function and signaling pathways of PTGFRN in HCC, GO and KEGG enrichment analyses of PTGFRN co-expressed genes were performed. A total of 215 co-expressed genes of PTGFRN were obtained by screening. As shown in Fig. 6A, PPI analysis using STRING database revealed that 10 genes, including PTGFR, CD81, CD9, TSPAN15, TSPAN2, CD63, CD82, GDPD5, CD151, and ENSG00000250349 have interacted with PTGFRN. KEGG analysis showed that PTGFRN co-expressed genes were mainly concentrated in the regulatory signaling pathways of "PI3K-Akt signaling pathway", "ECM-receptor interaction”, and "Regulation of actin cytoskeleton" (Fig. 6B). GO functional analysis showed that co-expressed genes were mainly involved in biological processes such as "extracellular matrix organization", "epithelial-mesenchymal transformation", "TNF-a/NF-kB signaling pathway”, "chemotaxis" and "angiogenesis” (Fig. 6C).

\section{Discussion}


Due to the cellular heterogeneity and complex pathogenesis of HCC, its fatality rate remains relatively high worldwide [20,21]. In recent years, more and more evidence have shown that prognostic gene markers have great potential in the prognosis of HCC [8]. In this study, the PTGFRN gene was used as the research object, and 377 HCC patients from TCGA were evaluated and found factors related to high expression of PTGFRN, namely clinical stage, histological grade, and survival time. The potential functions and related pathways of PTGFRN in HCC were obtained by enrichment analysis. It further confirmed the correlation between PTGFRN and the malignant phenotype and prognosis of HCC, and clarified the importance of PTGFRN as a potential biomarker of HCC.

In this study, we used the TCGA database for bioinformatics assessment to explore the expression level of PTGFRN in HCC. Previous studies have shown that PTGFRN is overexpressed in renal cell carcinoma and glioblastoma multiforme and is closely associated with poor prognosis [14, 22]. In addition, PTGFRN has also been shown to be increased in lung tumors and is closely associated with lung tumor growth restriction and lung transplantation metastatic status [15]. We can see that the gene PTGFRN is highly expressed in many cancers, and we found that PTGFRN is also highly expressed in HCC patients. In addition, we analyzed the relevant expression data and found that the expression of PTGFRN in HCC was significantly higher than that of adjacent non-tumor tissues. Therefore, we speculated that the high expression of PTGFRN might affect the related progression of HCC, and carried out further analysis.

Next, we performed a series of correlation analyses in order to explore whether PTGFRN could be used as an independent target. In our study, we discovered increased expression PTGFRN in HCC associated with latestage clinical and pathological features and predict a poor prognosis. The elevated expression level of PTGFRN at the high stage suggests a negative impact on the progression of HCC. Importantly, we found that the high expression of PTGFRN in tumor tissues is closely related to shorter OS and DFS. Global gene expression profile analysis of HCC tissue samples showed that PTGFRN mRNA expression was significantly up-regulated compared with normal liver tissue. The ROC curve of PTGFRN showed that its mRNA expression level had a high diagnostic value for HCC. Previous studies have not clarified the relationship between PTGFRN expression level and HCC tissue stage. However, the present study found that PTGFRN expression has a good predictive ability in low, high and histological grades. Through the analysis of HCC stage M and $\mathrm{N}$, although the results were not statistically significant, it was undeniable that the expression level of PTGFRN in $\mathrm{M} 1$ and $\mathrm{N} 1$ stages was higher than that in $\mathrm{M} 0$ and N0 stages. In addition, multivariate Cox analysis showed that overexpression of PTGFRN was an independent risk factor affecting the survival of patients with HCC. Therefore, according to the data we analyzed above, PTGFRN can serve as a potential new therapeutic target and an independent prognostic factor for HCC. Moreover, we urgently need to understand how the high expression of PTGFRN affects the occurrence and development of HCC.

To further explore the potential pathways regulated by PTGFRN in HCC, we performed GSEA analysis of PTGFRN. We have observed that the high expression phenotype of PTGFRN is mainly enriched to "endocytosis", "oocyte meiosis", "cancer pathways", "purine metabolism", "renal cell carcinoma", "regulation of actin cytoskeleton", "glioma" and "ERBB signaling pathway". Studies have pointed out that changes in the endocytosis of cancer cells will affect the changes in related cancer signaling pathways, thereby promoting the occurrence and development of tumors [23]. Zhou et al. [24] found that overexpression of SNX5 can 
promote the migration and invasion of HCC cells by mediating endocytosis. Qi et al. [25] found that CD147 was up-regulated in $\mathrm{HCC}$, and its endocytosis played a key role in the occurrence, invasion, and distant metastasis of HCC. We suspect that the overexpression of PTGFRN may affect the progression of HCC by mediating endocytosis. In addition, related study has shown that purine nucleotides are the basic and necessary factors of tumor cell proliferation, abnormal purine metabolism can promote the rapid proliferation and growth of tumor cells [26]. For cancer pathways, overexpression of miR-296-5p has been reported to inhibit the oncogenic role of the ERBB signaling pathway in HCC with a favorable prognosis [27]. In addition to the ERBB pathway, oocyte meiosis and jak/stat pathways have also been shown to play an important role in the carcinogenesis or progression of HCC $[28,29]$. Perhaps this prompted that HCC activates the various signaling pathway by increasing the expression of PTGFRN, which in turn leads to a poor prognosis for patients. GSEA results also showed that PTGFRN was involved in the regulation of the actin cytoskeleton. Actin regulatory proteins are considered to be a basic mechanism of cell migration. Studies have shown that actin regulatory proteins are considered to be the basic mechanism of cell migration. It is closely related to the poor prognosis of breast cancer and liver cancer, and is involved in the development of many cancers [30]. Based on GSEA, we also identified pathways associated with the cell cycle. However, the effect of transcriptional activation of PTGFRN on cell cycle progression and HCC progression still needs to be further studied. It can be seen that PTGFRN is related to many important pathways, and its up-regulation changes may affect the changes of a series of important pathways.

Finally, GO and KEGG enrichment analysis was performed on the co-expression genes of PTGFRN, and some important findings were obtained. The results showed that PTGFRN co-expressed genes were mainly involved in biological processes such as "PI3K-Akt signaling pathway", "ECM-receptor interaction", "extracellular matrix organization", "cell development", "angiogenesis", "adhesion", "TNF-a/NF-kB signaling pathway" and "epithelial-mesenchymal transformation". As we all know, the growth and migration of solid tumor cells are closely related to angiogenesis. S Colin et al. found that the domain GS-168AT2 of PTGFRN can inhibit the proliferation and migration of human endothelial cells ( $h E C$ ) in vitro and in vivo, and its anti-angiogenic activity is likely to be the main reason for the anti-tumor growth effect [15]. It indicated that PTGFRN might be involved in the malignant biological behavior of HCC. Moreover, our study also demonstrated that overexpression of PTGFRN was significantly associated with histological grade of HCC and predicted poor prognosis. In addition, the co-expression genes of PTGFRN are also importantly involved in the extracellular matrix organization (EMO) process. Previous studies have shown that EMO can provide a complex microenvironment for HCC cells and actively participate in the tumor development of HCC, such as angiogenesis, epithelial-mesenchymal transformation (EMT), invasion and metastasis [31, 32]. Besides, EMT is believed to play an important role in the anti-apoptosis, invasion, chemical drug resistance and other pathological processes of HCC cells [33, 34]. In addition, we found that PI3K-Akt and NF-kB signaling pathways were also significantly enriched. Studies have shown that NF-kB can act as downstream effectors of the PI3K-Akt pathway, and their abnormal activation is positively correlated with the malignant progression of HCC and leads to poor prognosis of HCC patients [35]. These enriched pathways suggest that PTGFRN may be involved in the progression of HCC at different stages. In addition, GO term enrichment analysis showed that PTGFRN may affect the cycle progression of tumor cells through cell adhesion and cell proliferation. Studies have confirmed that cell adhesion molecule overexpression can interact with ECM receptor, which is the key factor to accelerate the metastasis of HCC cells in blood vessels [36]. In addition, 
recent studies have shown that inhibition of PTGFRN can lead to a decrease in cell proliferation levels [14]. Furthermore, we reported for the first time that the expression of PTGFRN may also be related to chemotaxis, lateral plasma membrane, and inflammatory response pathways. But its regulatory mechanism needs to be further elucidated. In summary, the abundant GO and KEGG pathways explain the important role of PTGFRN in the progression of HCC to a certain extent.

\section{Conclusions}

In summary, our study preliminarily confirmed that PTGFRN is a promising biomarker for the diagnosis and prognosis of HCC. We also further explored its potential mechanisms in HCC. To our knowledge, this is the first study to determine the correlation between PTGFRN expression and clinical features of HCC based on database mining. However, this study was limited by its retrospective nature, and further studies are needed to confirm these results. Current studies on the functional role of PTGFRN are superficial, and future studies should focus on the molecular mechanisms by which PTGFRN promotes the occurrence and development of HCC.

\section{Materials \& Methods}

\section{Data Mining and Collection}

The corresponding mRNA sequencing data and clinical data of HCC patients were downloaded from TCGA. Samples with missing expression data were excluded from the study. Finally, the transcriptome sequencing (RNA-Seq) gene expression level 3 high-throughput sequencing - fragments per Kilobase of transcript per million mapped reads (HTSeq-FPKM) data of 377 patients with LIHC and clinical data were retained and further analyzed [37]. Obtain the corresponding RNA-Seq expression data of patients in the public openaccess database The Human Protein Atlas Project (http://v13.proteinatlas.org/) [38].

\section{Kaplan - Meier survival analysis}

Survival analysis was performed using gene expression profiling and interaction analysis (GEPIA) (http://gepia2.cancer-pku.cn/). Kaplan - Meier plots of overall survival (OS) and disease-free survival (DFS) were drawn and hazard ratios (HRs) were calculated for each selected gene individually. The $P$-value was calculated by log-rank test. The Cox proportional hazard ratio and the $95 \%$ confidence interval information can also be included in the survival plot [39]. The $P$-ROC package was used to draw the receiver operating characteristic curve (ROC) to evaluate the diagnostic value [40].

\section{Statistical analysis}

All acquired data were statistically analyzed by R (V.3.6.3). The relationship between clinicopathological characteristics and PTGFRN were analyzed with the Wilcoxon signed-rank test and logistic regression. Univariate Cox was used to analyzing and evaluate potential prognostic factors, and multivariate Cox analysis was used to compare the effect of PTGFRN expression on survival and other clinical characteristics (age, gender, stage, lymph node status, and distant metastasis). $P<0.05$ was considered statistically significant [41]. 


\section{Functional enrichment analysis}

Using The co-expression function of The cBioPortal for Cancer Genomics (http://cbioportal.org), sequencing data named hepatocellular carcinoma (TCGA, 373 cases) were selected for co-expression analysis of PTGFRN [42]. Protein-protein interaction analysis (PPI) PTGFRN was executed through the STRING database (https://string-db.org/) [43]. The genes with a Pearson S correlation greater than 0.4 were selected as coexpressed genes of PTGFRN. With DAVID system (https://david.ncifcrf.gov/) and gene ontology (GO) and genome encyclopedia (KEGG) terms of PTGFRN expressed genes for further analysis to determine the potential biological functions and methods. GO and KEGG enrichment analyzes were based on the $P$-value and q-value thresholds of $<0.05$ [44].

\section{Gene Set Enrichment Analysis (GSEA)}

GSEA is a computational approach that determines whether a set of a pre-defined genes show statistically significant differences between two biological phenotypes [45]. TCGA-LIHC RNA-seq data as gene expression data. We choose the annotated c2.cp.kegg.v7.2.symbols.gmt gene set as the reference gene set. The normalized enrichment score (NES) obtained by 1000 permutation analysis was used as the main statistic. Genes with false discovery rate (FDR) $<0.25$ and Nominal (NOM) $P$-value $<0.05$ were considered to be significantly enriched.

\section{Declarations}

Ethics approval and consent to participate

Not applicable.

\section{Consent for publication}

Not applicable.

\section{Competing interests}

The authors declare that they have no competing interests.

\section{Funding}

This work was supported by grants from the Guangxi Natural Science Foundation Project (No. 2020GXNSFBA297150 and 2018GXNSFAA138144), National Natural Science Foundation of China (81660464) and the Practice and Innovation Training Project for College Students in Guangxi Zhuang Autonomous Region (No. 202010598080).

\section{Competing Interests}

The authors declare that they have no competing interests.

\section{Author contributions}


Conception and design: QNZ and NXC. Information of database collection: YLT and YTL. Statistical analysis: YLT, FW and YQW. Manuscript writing: YLT, WZL and QNZ. Final approval of manuscript: All authors.

\section{Acknowledgements}

The authors would like to thank the National Natural Science Foundation of China, the Guangxi Natural Science Foundation Project and the Practice and Innovation Training Project for College Students in Guangxi Zhuang Autonomous Region.

\section{Availability of data and material}

The following information was supplied regarding data availability:

The datasets analyzed in this study are available in the following open access

repositories:

The Cancer Genome Atlas (TCGA), https://cancergenome.nih.gov/.

The Human Protein Atlas Project, http://v13.proteinatlas.org/

Gene Expression Profiling and Interaction Analysis (GEPIA), http://gepia2.cancer-pku.cn/

STRING database, https://string-db.org/

The Database for Annotation, Visualization and Integrated Discovery (DAVID ), https://david.ncifcrf.gov/

The raw measurements are available in the Supplemental Files.

\section{References}

1. Jiao Y, Li Y, Jia B, Chen Q, Pan G, Hua F, et al. The prognostic value of IncRNA SNHG4 and its potential mechanism in liver cancer. Biosci Rep. 2020;40(1).

2. Llovet JM, Zucman-Rossi J, Pikarsky E, Sangro B, Schwartz M, Sherman M, et al. Hepatocellular carcinoma. Nature Reviews Disease Primers. 2016;2(1):16018.

3. Qin G, Su J, Ning Y, Duan X, Luo D, Lotlikar PD. p53 protein expression in patients with hepatocellular carcinoma from the high incidence area of Guangxi, Southern China. Cancer Lett. 1997;121(2):203-10.

4. Chaudhary K, Poirion OB, Lu L, Garmire LX. Deep Learning-Based Multi-Omics Integration Robustly Predicts Survival in Liver Cancer. Clinical Cancer Research. 2018;24(6):1248-59.

5. Lurje I, Czigany Z, Bednarsch J, Roderburg C, Isfort P, Neumann UP, et al. Treatment Strategies for Hepatocellular Carcinoma (-) a Multidisciplinary Approach. Int J Mol Sci. 2019;20(6).

6. Liu Y, Yang Y, Luo Y, Wang J, Lu X, Yang Z, et al. Prognostic potential of PRPF3 in hepatocellular carcinoma. Aging (Albany NY). 2020;12(1):912-30.

7. Zhang FP, Huang YP, Luo WX, Deng WY, Liu CQ, Xu LB, et al. Construction of a risk score prognosis model based on hepatocellular carcinoma microenvironment. World J Gastroenterol. 2020;26(2):134-53. 
8. Liu GM, Zeng HD, Zhang CY, Xu JW. Identification of a six-gene signature predicting overall survival for hepatocellular carcinoma. Cancer Cell Int. 2019;19:138.

9. Bruix J, Reig M, Sherman M. Evidence-Based Diagnosis, Staging, and Treatment of Patients With Hepatocellular Carcinoma. Gastroenterology. 2016;150(4):835-53.

10. Marrero JA, Feng Z, Wang Y, Nguyen MH, Befeler AS, Roberts LR, et al. Alpha-fetoprotein, des-gamma carboxyprothrombin, and lectin-bound alpha-fetoprotein in early hepatocellular carcinoma. Gastroenterology. 2009;137(1):110-8.

11. Long J, Zhang L, Wan X, Lin J, Bai Y, Xu W, et al. A four-gene-based prognostic model predicts overall survival in patients with hepatocellular carcinoma. J Cell Mol Med. 2018;22(12):5928-38.

12. Colin S, Guilmain W, Creoff E, Schneider C, Steverlynck C, Bongaerts M, et al. A truncated form of CD9partner 1 (CD9P-1), GS-168AT2, potently inhibits in vivo tumour-induced angiogenesis and tumour growth. Br J Cancer. 2011;105(7):1002-11.

13. Andre M, Chambrion C, Charrin S, Soave S, Chaker J, Boucheix C, et al. In situ chemical cross-linking on living cells reveals CD9P-1 cis-oligomer at cell surface. J Proteomics. 2009;73(1):93-102.

14. Aguila B, Morris AB, Spina R, Bar E, Schraner J, Vinkler R, et al. The Ig superfamily protein PTGFRN coordinates survival signaling in glioblastoma multiforme. Cancer Lett. 2019;462:33-42.

15. Guilmain W, Colin S, Legrand E, Vannier JP, Steverlynck C, Bongaerts M, et al. CD9P-1 expression correlates with the metastatic status of lung cancer, and a truncated form of CD9P-1, GS-168AT2, inhibits in vivo tumour growth. Br J Cancer. 2011;104(3):496-504.

16. Stegeman H, Span PN, Kaanders JH, Bussink J. Improving chemoradiation efficacy by PI3-K/AKT inhibition. Cancer Treat Rev. 2014;40(10):1182-91.

17. Sanchez-Rodriguez R, Torres-Mena JE, De-la-Luz-Cruz M, Bernal-Ramos GA, Villa-Trevino S, ChagoyaHazas V, et al. Increased expression of prostaglandin reductase 1 in hepatocellular carcinomas from clinical cases and experimental tumors in rats. Int J Biochem Cell Biol. 2014;53:186-94.

18. Zhang M, Zhang H, Cheng S, Zhang D, Xu Y, Bai X, et al. Prostaglandin E2 accelerates invasion by upregulating Snail in hepatocellular carcinoma cells. Tumour Biol. 2014;35(7):7135-45.

19. Yuan JM, Grouls M, Carmella SG, Wang R, Heskin A, Jiang Y, et al. Prediagnostic levels of urinary 8-epiprostaglandin F2alpha and prostaglandin E2 metabolite, biomarkers of oxidative damage and inflammation, and risk of hepatocellular carcinoma. Carcinogenesis. 2019;40(8):989-97.

20. Bruix J, Gores GJ, Mazzaferro V. Hepatocellular carcinoma: clinical frontiers and perspectives. Gut. 2014;63(5):844-55.

21. Li WC, Xiong ZY, Huang PZ, Liao YJ, Li QX, Yao ZC, et al. KCNK levels are prognostic and diagnostic markers for hepatocellular carcinoma. Aging (Albany NY). 2019;11(19):8169-82.

22. Huang G, Lai Y, Pan X, Zhou L, Quan J, Zhao L, et al. Tumor suppressor miR-33b-5p regulates cellular function and acts a prognostic biomarker in RCC. Am J Transl Res. 2020;12(7):3346-60.

23. Schmid SL. Reciprocal regulation of signaling and endocytosis: Implications for the evolving cancer cell. J Cell Biol. 2017;216(9):2623-32.

24. Zhou Q, Huang T, Jiang Z, Ge C, Chen X, Zhang L, et al. Upregulation of SNX5 predicts poor prognosis and promotes hepatocellular carcinoma progression by modulating the EGFR-ERK1/2 signaling 
pathway. Oncogene. 2020;39(10):2140-55.

25. Qi S, Su L, Li J, Zhao P, Zhang Q, Niu X, et al. YIPF2 is a novel Rab-GDF that enhances HCC malignant phenotypes by facilitating CD147 endocytic recycle. Cell Death Dis. 2019;10(6):462.

26. Yin J, Ren W, Huang X, Deng J, Li T, Yin Y. Potential Mechanisms Connecting Purine Metabolism and Cancer Therapy. Front Immunol. 2018;9:1697.

27. Shi DM, Li LX, Bian XY, Shi XJ, Lu LL, Zhou HX, et al. miR-296-5p suppresses EMT of hepatocellular carcinoma via attenuating NRG1/ERBB2/ERBB3 signaling. J Exp Clin Cancer Res. 2018;37(1):294.

28. Calvisi DF, Ladu S, Gorden A, Farina M, Conner EA, Lee JS, et al. Ubiquitous activation of Ras and Jak/Stat pathways in human HCC. Gastroenterology. 2006;130(4):1117-28.

29. Wang Z, Xu J, Zhang S, Chang L. Expression of cell divisioncycle-associated genes and their prognostic significance in hepatocellular carcinoma. Int J Clin Exp Pathol. 2021;14(2):151-69.

30. Iwaya K, Norio K, Mukai K. Coexpression of Arp2 and WAVE2 predicts poor outcome in invasive breast carcinoma. Mod Pathol. 2007;20(3):339-43.

31. Novikova MV, Khromova NV, Kopnin PB. Components of the Hepatocellular Carcinoma Microenvironment and Their Role in Tumor Progression. Biochemistry (Mosc). 2017;82(8):861-73.

32. Zhang Y, Mao X, Chen W, Guo X, Yu L, Jiang F, et al. A Discovery of Clinically Approved Formula FBRP for Repositioning to Treat HCC by Inhibiting PI3K/AKT/NF-kappaB Activation. Mol Ther Nucleic Acids. 2020;19:890-904.

33. Qi LN, Xiang BD, Wu FX, Ye JZ, Zhong JH, Wang YY, et al. Circulating Tumor Cells Undergoing EMT Provide a Metric for Diagnosis and Prognosis of Patients with Hepatocellular Carcinoma. Cancer Res. 2018;78(16):4731-44.

34. Chen L, Guo P, He Y, Chen Z, Chen L, Luo Y, et al. HCC-derived exosomes elicit HCC progression and recurrence by epithelial-mesenchymal transition through MAPK/ERK signalling pathway. Cell Death Dis. 2018;9(5):513.

35. Chen H, Wang H, Yu X, Zhou S, Zhang Y, Wang Z, et al. ERCC6L promotes the progression of hepatocellular carcinoma through activating PI3K/AKT and NF-kappaB signaling pathway. BMC Cancer. 2020;20(1):853.

36. Zhang C, Peng L, Zhang Y, Liu Z, Li W, Chen S, et al. The identification of key genes and pathways in hepatocellular carcinoma by bioinformatics analysis of high-throughput data. Med Oncol. 2017;34(6):101.

37. Deng X, Jiang Q, Liu Z, Chen W. Clinical Significance of an m6A Reader Gene, IGF2BP2, in Head and Neck Squamous Cell Carcinoma. Front Mol Biosci. 2020;7:68.

38. Uhlen M, Zhang C, Lee S, Sjostedt E, Fagerberg L, Bidkhori G, et al. A pathology atlas of the human cancer transcriptome. Science. 2017;357(6352).

39. Li X, Yu W, Liang C, Xu Y, Zhang M, Ding X, et al. INHBA is a prognostic predictor for patients with colon adenocarcinoma. BMC Cancer. 2020;20(1):305.

40. Zhang Z, Wang S, Liu Y, Meng Z, Chen F. Low IncRNA ZNF385DAS2 expression and its prognostic significance in liver cancer. Oncol Rep. 2019;42(3):1110-24. 
41. Wu H, Zhang J. Decreased expression of TFAP2B in endometrial cancer predicts poor prognosis: A study based on TCGA data. Gynecol Oncol. 2018;149(3):592-7.

42. Cerami E, Gao J, Dogrusoz U, Gross BE, Sumer SO, Aksoy BA, et al. The cBio cancer genomics portal: an open platform for exploring multidimensional cancer genomics data. Cancer Discov. 2012;2(5):401-4.

43. Shi J, Chen L, Chen Y, Lu Y, Chen X, Yang Z. Aldo-Keto Reductase Family 1 Member B10 (AKR1B10) overexpression in tumors predicts worse overall survival in hepatocellular carcinoma. $J$ Cancer. 2019;10(20):4892-901.

44. Lin W, Chen X, Chen T, Liu J, Ye Y, Chen L, et al. C1QTNF6 as a Novel Diagnostic and Prognostic Biomarker for Clear Cell Renal Cell Carcinoma. DNA Cell Biol. 2020;39(6):1000-11.

45. Hu B, Yang XB, Sang XT. Development of an immune-related prognostic index associated with hepatocellular carcinoma. Aging (Albany NY). 2020;12(6):5010-30.

\section{Figures}

A

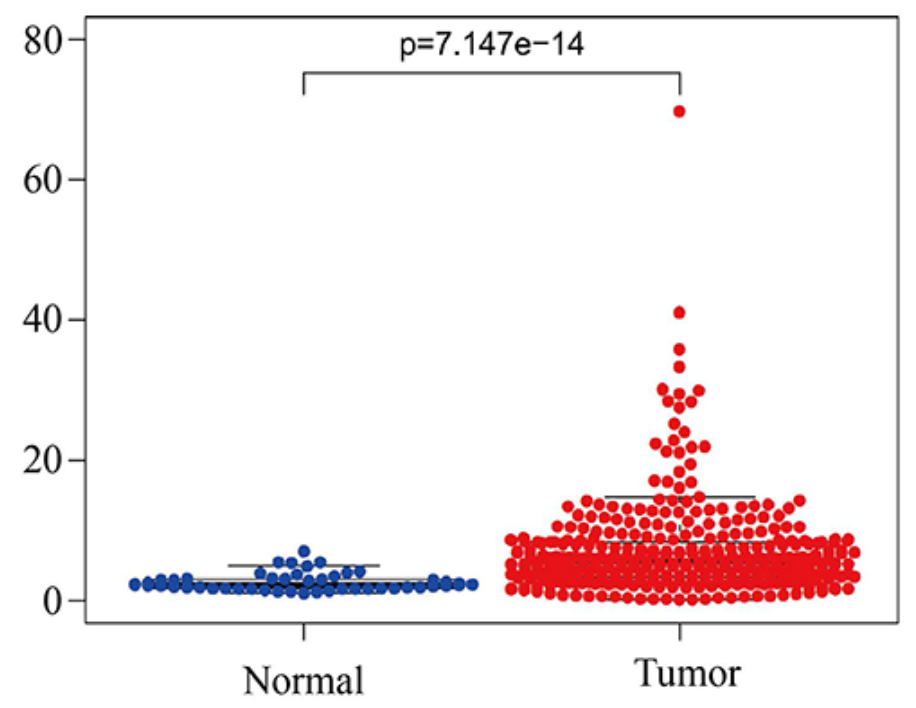

B

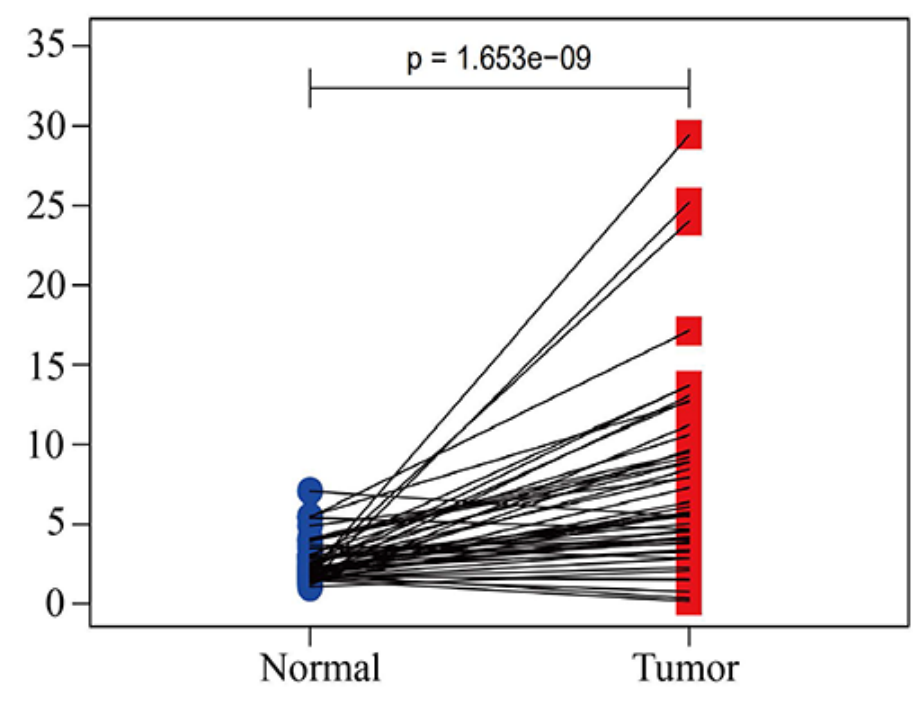

C

\section{PTGFRN}

$4 \times$

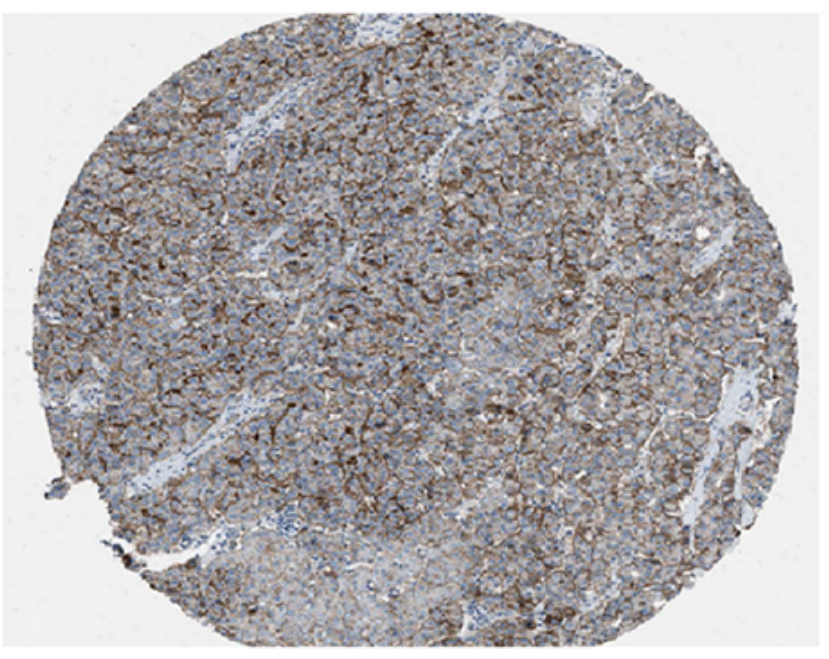

$4 \times$

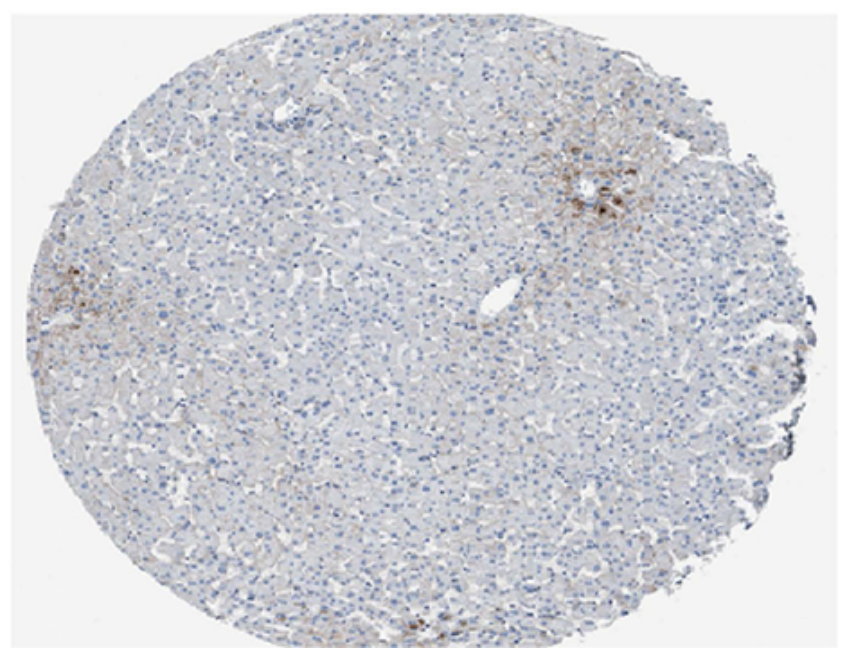


Figure 1

PTGFRN expression in HCC. A: PTGFRN in 374 cases of HCC tissues and the general expression in 50 cases of tissue adjacent to carcinoma; B: Paired difference analysis based on PTGFRN mRNA expression in the TCGA - LIHC cohort; C: Immunohistochemical results showed low expression of PTGFRN in adjacent tissues (4×); D: High expression of PTGFRN in HCC tissues (4x)

A

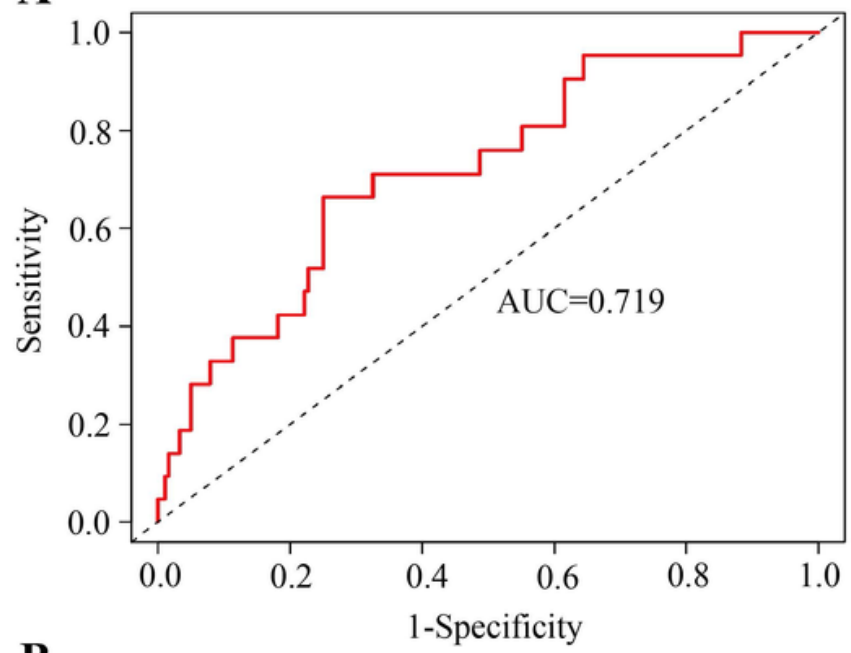

B
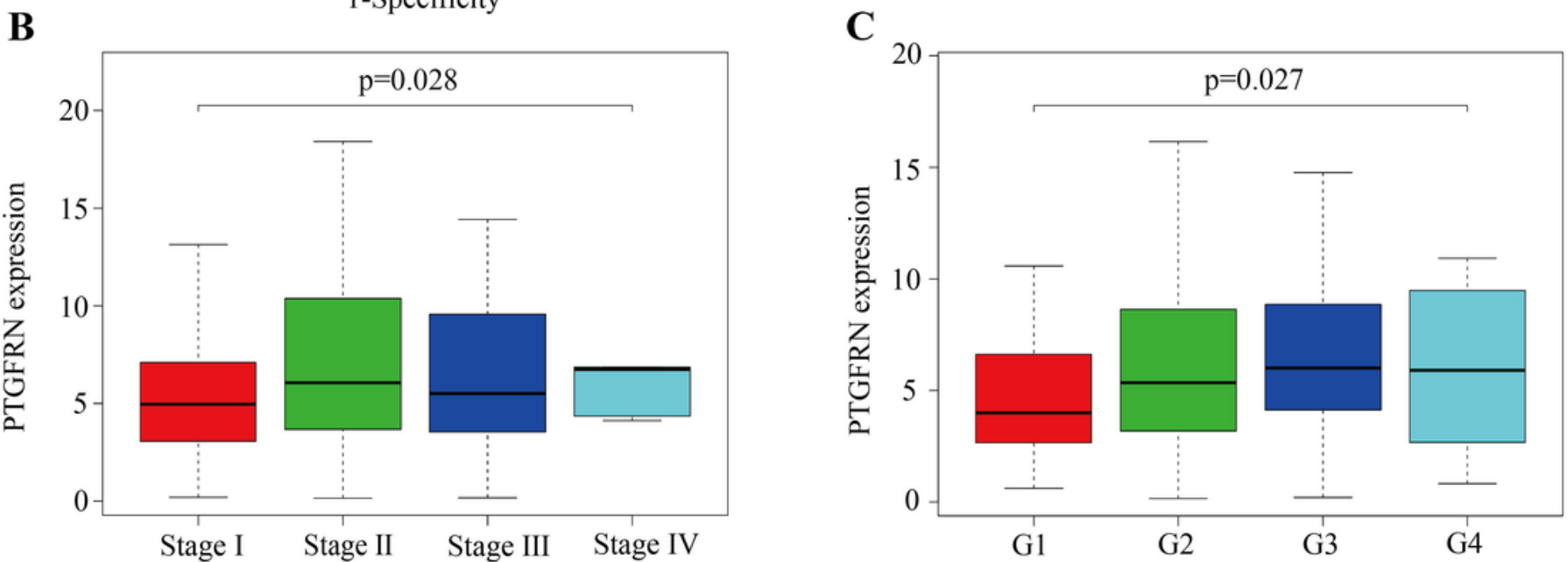

D
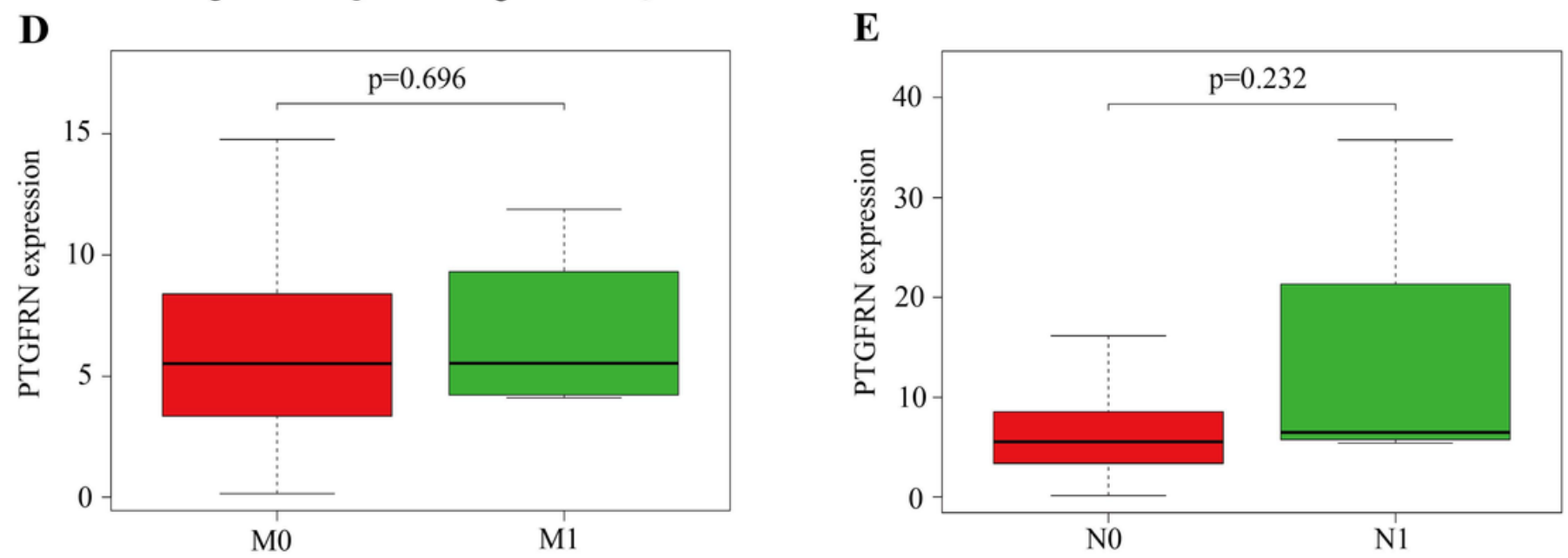

Figure 2 
TCGA database analysis of the relationship between PTGFRN and clinical and pathological parameters. A: ROC curve verifies the diagnostic value of up-regulation of PTGFRN expression in HCC B: PTGFRN expression in clinical stages of HCC patients; C: The expression of PTGFRN in the histological grading of HCC patients; D: The expression of PTGFRN in distant metastasis of HCC patients; E: Expression of PTGFRN in lymph node metastasis in HCC patients

A

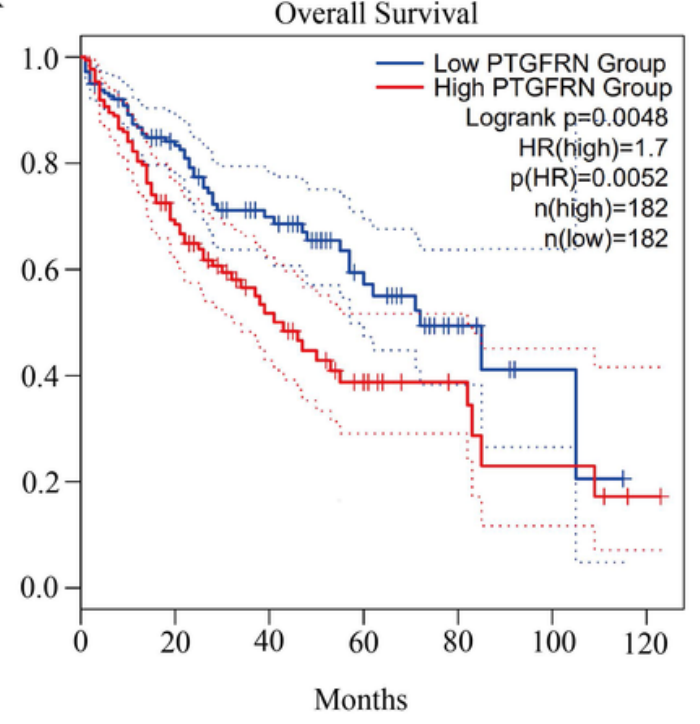

B

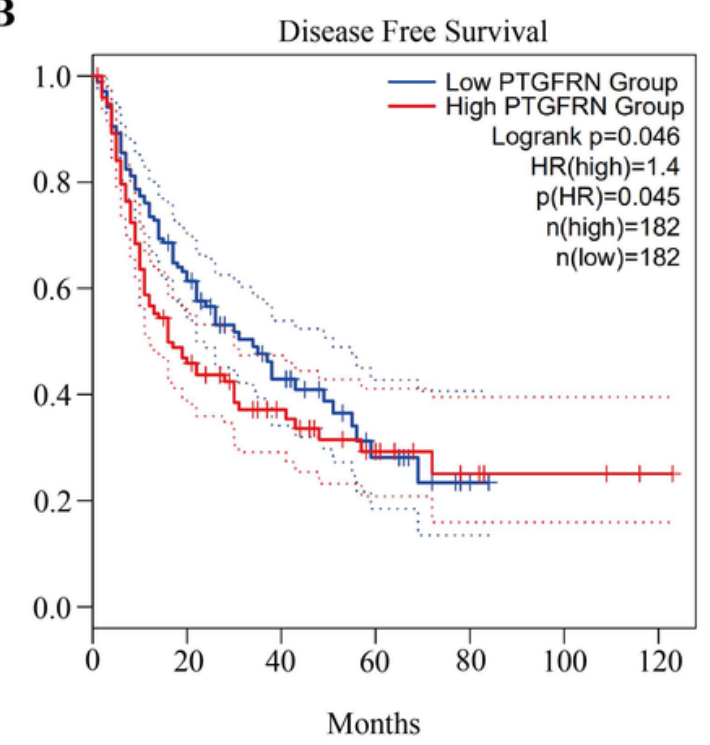

C

Hazard ratio

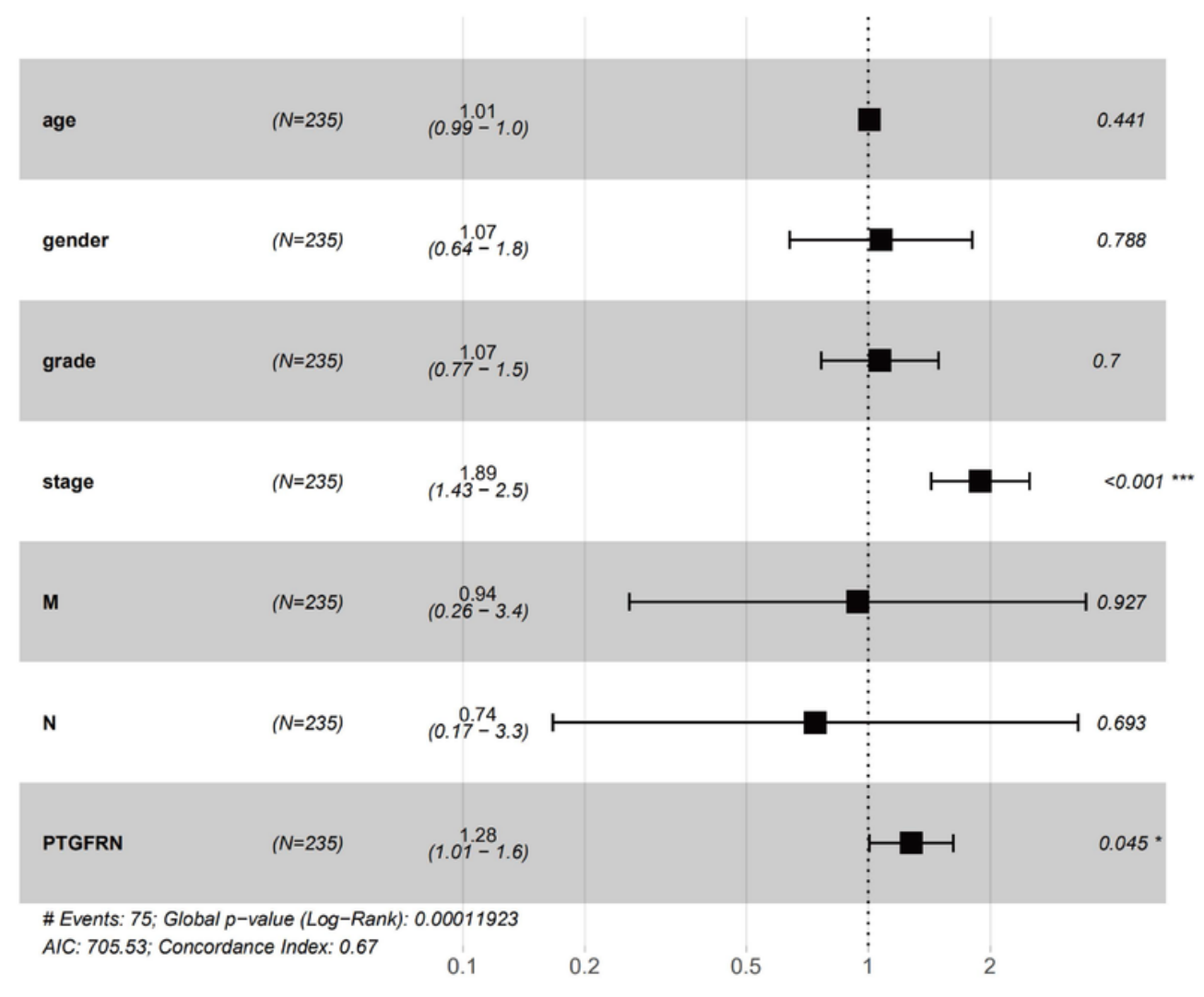

Figure 3 
Survival analysis and forest map analysis. A: Expression of PTGFRN and survival analysis of overall survival; B: Expression of PTGFRN and survival analysis of disease-free survival; C: Expression and clinicopathological characteristics of forest atlases

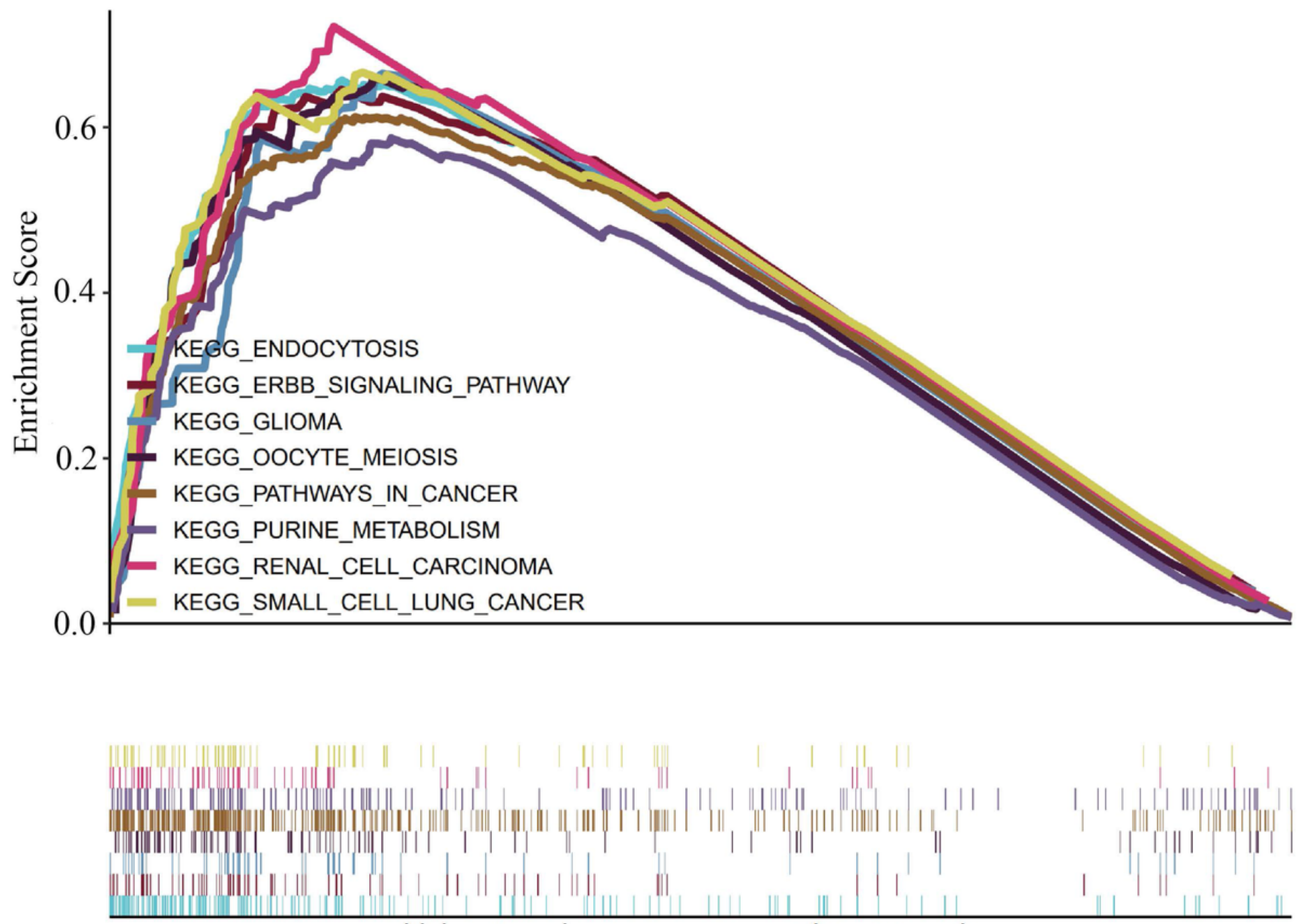

high expression<------------->low expression

Figure 4

Concentration graph of multiple GSEA enrichment pathways 
A

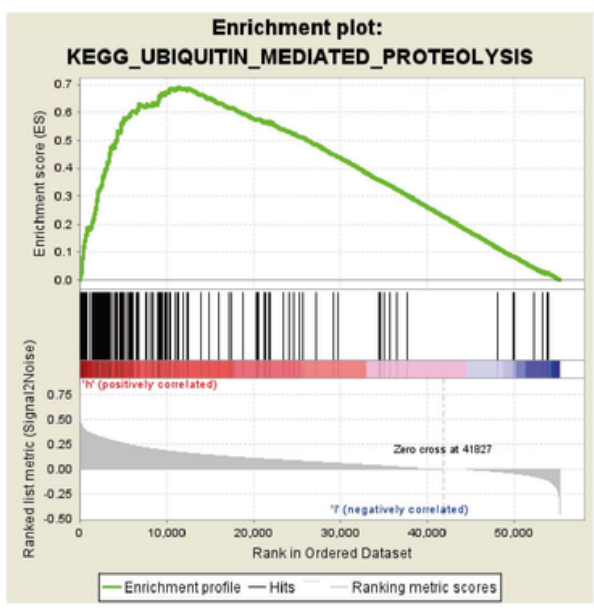

D

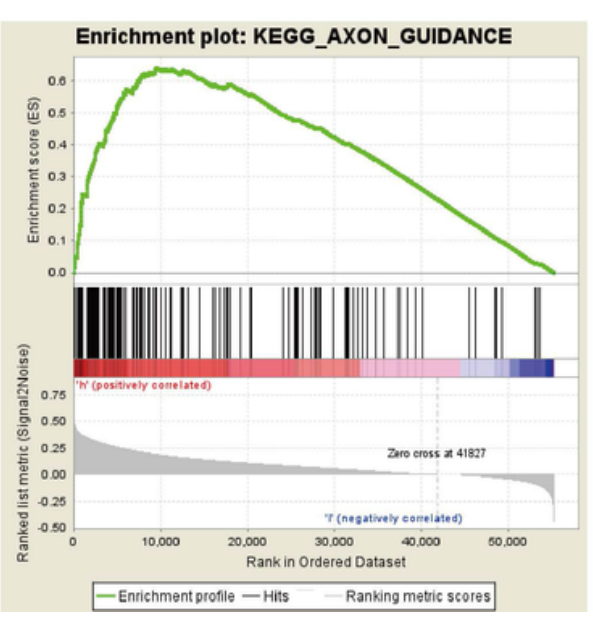

B

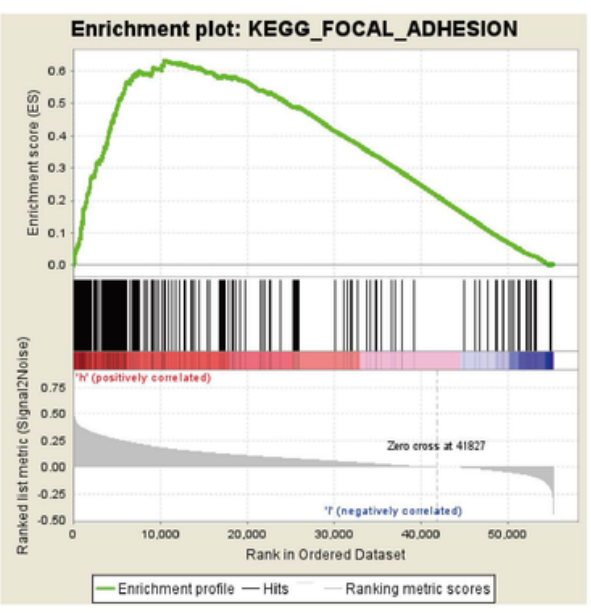

$\mathbf{E}$

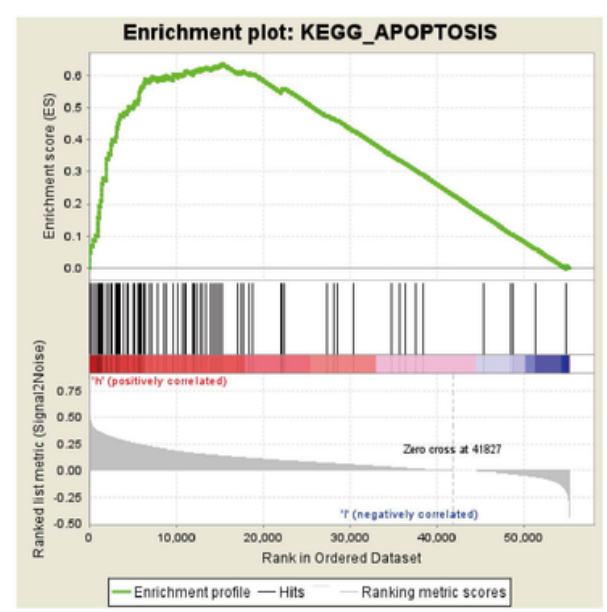

C

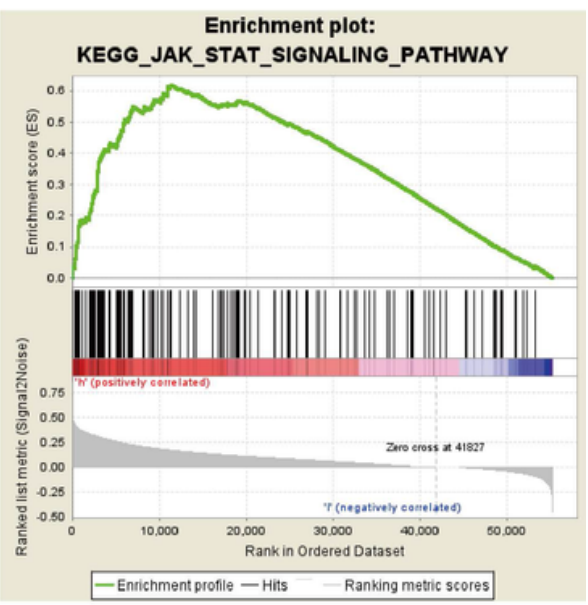

F

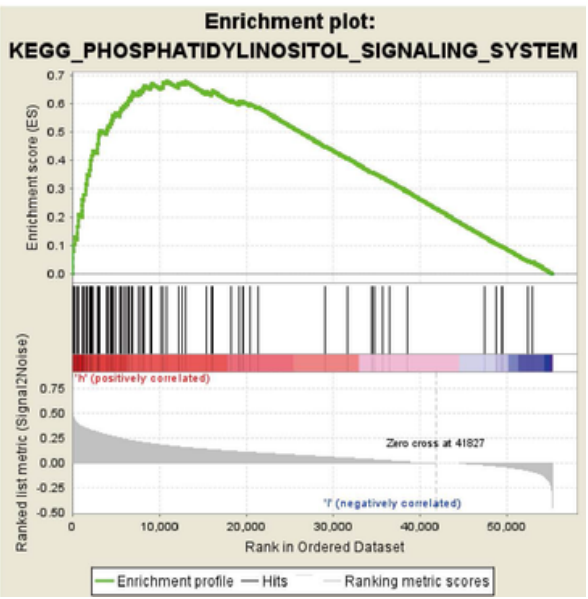

\section{Figure 5}

Pathways associated with GSEA enrichment. A-F: The results showed that "ubiquitin-mediated proteolysis", "adhesion plaque", "axon-directed", "apoptosis", "jak stat" and "phosphatidylinositol" signaling pathways were significantly enriched in the high expression phenotype of PTGFRN 
A

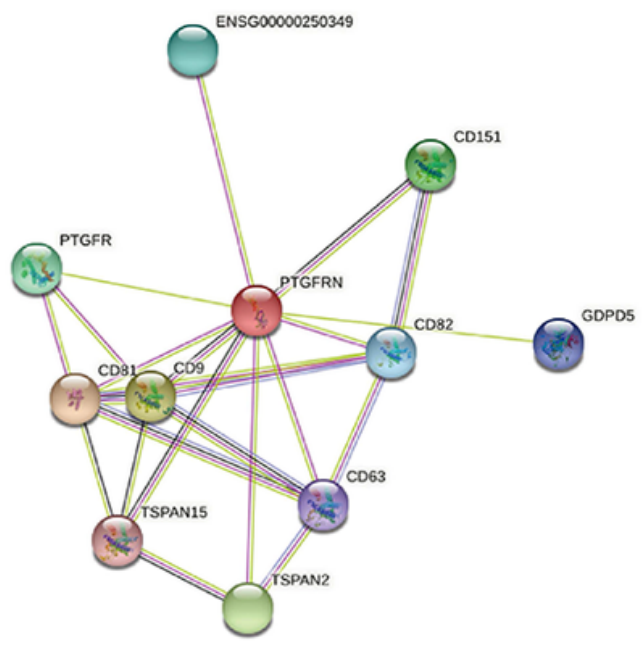

B

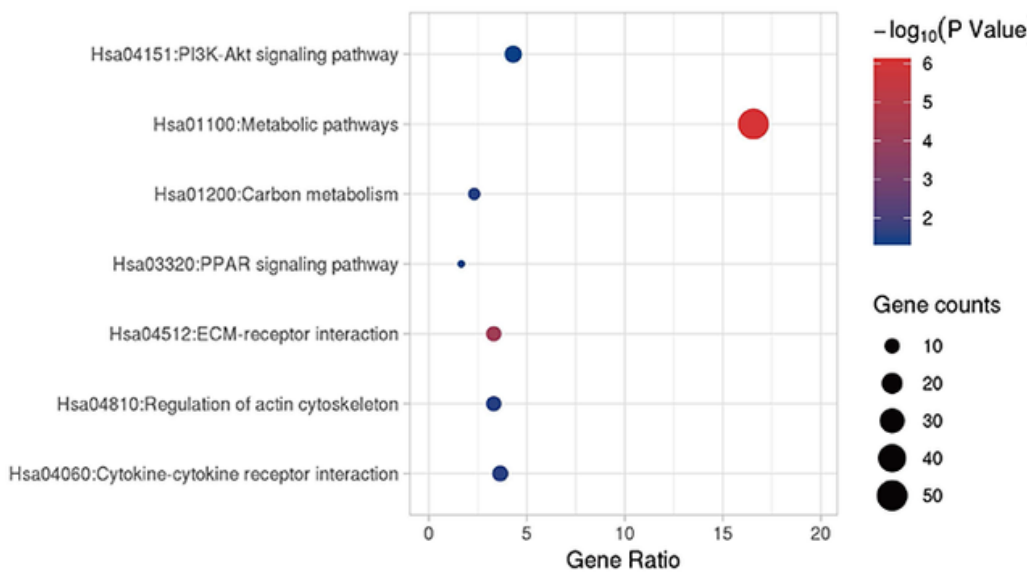

C

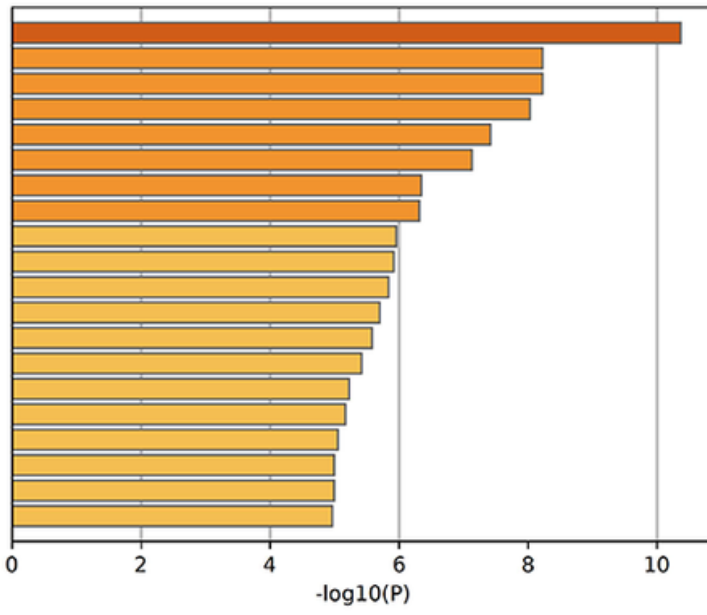

GO:0030198: extracellular matrix organization

M5930: HALLMARK EPITHELIAL MESENCHYMAL TRANSITION M5890: HALLMARK TNFA SIGNALING VIA NFKB GO:0006935: chemotaxis

GO:0016328: lateral plasma membrane

GO:0030155: regulation of cell adhesion

CORUM:2352: ITGAV-ITGB6-SPP1 complex

M5932: HALLMARK INFLAMMATORY RESPONSE

GO:0002274: myeloid leukocyte activation

GO:0048729: tissue morphogenesis

GO:0005126: cytokine receptor binding

GO:0007160: cell-matrix adhesion

GO:0022411: cellular component disassembly

GO:0016052: carbohydrate catabolic process

GO:0001525: angiogenesis

GO:0051046: regulation of secretion

GO:0071438: invadopodium membrane

GO:0010720: positive regulation of cell development

GO:0060135: maternal process involved in female pregnancy

GO:0030036: actin cytoskeleton organization

\section{Figure 6}

PPI interaction analysis and functional enrichment analysis. A: PTGFRN protein interaction network diagram in String; B: Biological process enrichment of KEGG co-expressed genes; C: Biological process enrichment of GO co-expressed gene 